\title{
Gluten-free diet — remedy for infertility or dangerous trend?
}

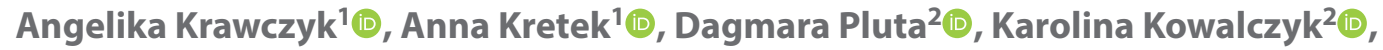

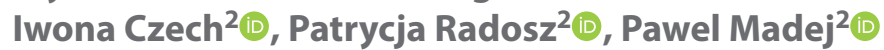 \\ 'Students Scientific Association of Gynecological Endocrinology, School of Medicine in Katowice, Medical University of Silesia, \\ Katowice, Poland \\ ${ }^{2}$ Department of Gynecological Endocrinology, School of Medicine in Katowice, Medical University of Silesia, Katowice, Poland
}

\begin{abstract}
Association between celiac disease (CD) and infertility has been a point of consideration for many years. There are lots of studies which present contradictory results. It is accepted that women with unexplained infertility have three times higher risk of $C D$ compared to the healthy women. This finding leads to the conclusion that a gluten-free diet (GFD), as the only effective method of treatment $C D$, is important to improve fertility. It is observed that women diagnosed with $\mathrm{CD}$ and treated by GFD, have positive pregnancy outcomes including better effects of assisted reproductive technology (ART). Excluding gluten from diet by women without CD diagnosis could bring adverse outcomes, because the risk of malnutrition if diet is unbalanced.

On the other hand, some research show that not only GFD but CD is related to malnutrition which could be the cause of problems with getting pregnant or with termination of pregnancy. Regarding CD influence on women's reproductive system, it is not only connected with unexplained infertility and adverse pregnancy outcomes, but also with delayed menarche, early menopause and decrease of anti-Müllerian hormone (AMH). What is more, non-celiac wheat sensitivity affects the reproductive system by increasing risk of menstrual disorders too.

The main aim of this paper is presenting scientific facts about the link between gluten elimination and fertility. It is very important to provide patients with reliable fact-checked information in the context of their symptoms.
\end{abstract}

Key words: celiac disease; infertility; reproductive disorders; gluten-free diet; adverse pregnancy outcomes

Ginekologia Polska 2022; 93, 5: 422-426

\section{INTRODUCTION}

Nowadays, increased popularity of different elimination diets is observed. Products with "gluten free", "lactose free" and similar labels are available in almost every store. Moreover, a lot of information about beneficial effects of these diets can be found in the popular media.

One of the common hypotheses is a relationship between $C D$ and infertility. This is the reason why information can be found that GFD facilitates getting pregnant. Lactose, sugar but also gluten elimination is considered to improve fertility. In social media, especially in some supporting groups for women with reproduction problems, women share their experience about improving hormonal results, well being and getting pregnant after diet modification.
In this paper we are trying to answer the question, if the gluten free diet is justified in gynaecological disorders including infertility.

\section{CELIAC DISEASE}

$\mathrm{CD}$ is one of the most common autoimmune diseases, consisting of producing autoantibodies in response to gluten ingestion, which is a component of products like wheat, rye and barley. The effect of their action is villous atrophy. The prevalence of $C D$ is different and depends on the geographic region, but it is estimated that in the general population it is approximately $1 \%$ [1]. The clinical picture is varied. The most common gastrointestinal symptoms are: diarrhoea, bloating and stomach ache. Sometimes typical

\footnotetext{
Corresponding author:

Angelika Krawczyk

Students Scientific Association of Gynecological Endocrinology, School of Medicine in Katowice, Medical University of Silesia, Katowice, Poland

e-mail: angelika.krawczyk328@gmail.com
} 
gastric symptoms do not appear and only one manifestation of the disease can be osteoporosis, neurological disorders, dermatology problems, anaemia or infertility [1]. The first line of diagnosis of $C D$ is designation of antibodies to tissue transglutaminase (TTG) and endomysial antibodies (EMA). False negative results can be obtained when a patient is on the GFD or suffers from IgA deficiency. The second line is histologic analysis of small bowel biopsies samples. Additionally, it is possible to confirm the diagnosis by genetic tests for alleles HLA-DQ2 and/or HLA-DQ8 [1]. It is necessary to remember that a positive result of these tests is not a confirmation of $C D$, but a negative result excludes the risk of this condition. An important fact is that CD is a completely curable disease, and the treatment consists of elimination of gluten from diet.

\section{CELIAC DISEASE AND FERTILITY}

According to the World Health Organisation, the definition of infertility is a failure to conceive after 12 months of regular unprotected intercourses [2]. It is estimated that $8-12 \%$ of couples in the world have reproductive problems [2]. What is significant, approximately $30 \%$ of them are diagnosed with unexplained infertility, which means that there is no apparent cause for this condition [2].

For the first time the relation between CD and infertility was described in 1970 by Morris [3]. Since that time, there have been new reports which try to contradict or confirm the association between these two conditions.

The connection of autoantibodies and reproduction problems is a very interesting and still analyzed issue [4-6]. It is proved that dysfunction of ovaries and even premature ovarian insufficiency occurs more often in women with autoimmunization [4, 5]. It all explains the interests in understanding how $\mathrm{CD}$, which is an autoimmune condition, impacts fertility. Also, macro- and micronutrients deficiency could be the reason for reproduction problems in CD [7]. The elements and vitamins with most common deficiency in this condition are iron (Fe), zinc ( $\mathrm{Zn})$, calcium, (Ca) vitamin D, vitamin B12 and folic acid [8]. Lower plasma zinc concentration in women is correlated with longer time to pregnancy [9]. It needs to be emphasised that zinc is an important element for the good semen quality. Based on those facts a hypothesis can be made that CD impacts also men's fertility $[9,10]$. Another crucial element is folic acid which is essential for proper cell divisions. Its deficiency could be a cause of anovulation and short menstrual cycles [11]. The best described deficiency in CD is iron deficiency resulting in anaemia. If a patient suffers from iron deficiency anaemia and reproduction problems serological assays for CD should be performed [12].

Because of contradictory data presented by different authors, in 2019 meta-analysis was published, whose main aim was to resume publications in this topic and make conclusions [13]. Results show approximately three times more frequent TTG and EMA antibodies in women with infertility [13]. The most significant group among them are women with unexplained infertility [13]. Data show that women suffering from CD but on GFD have the same risk of infertility and adverse pregnancy outcomes like the general population $[13,14]$. What is important, authors indicate that studies about association between CD and infertility use low sample size, which makes them insufficient to be base for recommendations. Another reason for absence of routine tests for $C D$ in infertility diagnostics are papers, which deny the existence of associations of these two conditions. Examples are Canadian [15] and Danish [16] papers. It is worth noting that despite many Danish studies about CD and reproduction problems, the prevalence of $C D$ in this geographic region is lower than in the general population (approx. 0.5\%).

It seems to be optimal to use point-of-care tests for CD antibodies in women with reproductive problems. Those tests are successfully used in different fields for a long time $[17,18]$. Studies confirm their accuracy and emphasize economic benefits compared to classic serologic assays $[17,18]$. One of them was checked for accuracy in infertility diagnostics. Unfortunately, its sensitivity was too low for this purpose [19]. However, it is a very promising direction and maybe, in the future, it will be possible to use this form of screening.

In literature some authors point out a link between untreated CD and delayed menarche [20] and early menopause [7]. A shorter reproductive period is a consequence. When a patient has a history of delayed menarche it can be suspected that the reason for her problems is CD [20]. Early menopause in case of $C D$ could be associated with decrease of $\mathrm{AMH}$, and in result decrease in ovarian reserve compared to healthy women [21]. It is worth mentioning that in the study which indicates correlation between decrease of $\mathrm{AMH}$ and $C D$, there is no information about using GFD in the analyzed group [21]. In conclusion, there is no information about the influence gluten intake has on AMH concentration. It has not been proved that excluding gluten from diet improves ovarian reserve. The research in this field is not robust enough to draw any conclusions which could motivate to further expand the analysis. It is worth knowing that $C D$ is not the only one autoimmune condition which results in the decrease of $\mathrm{AMH}$ [22].

\section{CELIAC DISEASE AND ADVERSE PREGNANCY OUTCOMES}

Recurrent spontaneous miscarriage is defined as a minimum of three miscarriages in the history of a patient, and according to some sources, even two miscarriages allow such a diagnosis to be made [23]. This group of patients is 
very often distinguished in the studies about the connection between $C D$ and fertility. It is most likely caused by the presence of transglutaminase, against which antibodies are present in patients with $C D$, on the microvilli of the syncytiotrophoblast [24]. The consequence is impaired development and function of the placenta, which may result not only in miscarriages but also in a low birth weight of newborns or intrauterine growth retardation (IUGR) [7, 14].

The second issue that strengthens the study of the above relationship is the aforementioned micronutrient deficiencies in CD. It has been known for many years that a mother's malnutrition in the early stages of pregnancy can result in its loss [9, 25-27]. Studies conducted on pregnant patients with a history of spontaneous miscarriages showed a reduced level of $\mathrm{Zn}, \mathrm{Cu}$, selenium and vitamin $\mathrm{E}$ compared to the control group $[25,26]$. Moreover, the low level of $\mathrm{Zn}$ in the maternal serum also correlates with the occurrence of preterm deliveries and preterm premature rupture of membranes (pPROM) [27]. A study about the influence of GFD on pregnancy outcomes in women suffering from $C D$ has been published recently. The study showed that following a GFD after diagnosing the disease is associated with more live births [28].

Although the arguments presented above explain how $C D$ may affect the occurrence of recurrent spontaneous miscarriages or one miscarriage, this relationship has not been clearly demonstrated in studies [13]. The reason may be that in most cases CD is analyzed among other factors when considering unexplained infertility. This results in the small sample size of the study group. Additionally, in existing studies, CD is only diagnosed based on serological tests, which also reduces the reliability of the results [13]. Although the research does not confirm the validity of screening for CD in women with recurrent spontaneous miscarriages, there is no doubt that women diagnosed with the disease should be instructed on the importance of adhering to a strict, but well-balanced GFD supplemented with the necessary elements, preferably under the control of a dietitian, for the maintenance of pregnancy and the birth of a healthy child.

The important question is whether anti-TTG antibodies could obstruct the formation of the placenta, causing infertility in women with CD [24]. Aside from the obvious problem with getting pregnant naturally, it would be worth considering what effect these autoantibodies have on the success of ART. Due to the fact that until now only few studies have been conducted on the impact of $C D$ on the success of ART, no definite conclusions can be drawn. In 2018 a study conducted on a group of 1,000 patients of the fertility clinic was published [29]. Its results clearly indicated no statistically significant difference in the number of live births as a result of in vitro fertilization (IVF) between women with CD and without it. The second con- clusion from this study was the effect of a GFD on IVF, and here the authors also found no difference in success between the gluten-free group and the control group [29, 30]. It should be mentioned, however, that in this study, CD was diagnosed only based on anti-TTG and anti-EMA antibodies, without histopathological examination. In addition, information about the use of a GFD was obtained through a questionnaire, and people declaring it did not have previously diagnosed CD. For this reason, it should be noted that the conclusion that the GFD does not affect IVF applies to its use by the general population and not by women with CD. In opposition to the above study, another one was published, in which seropositive patients had a small bowel biopsy and the ART results were analyzed before and after eliminating gluten from the diet [28]. This time, an increase in both the achieved pregnancies and live births was observed in women after the introduction of a GFD. Analyzing both studies at the same time, it can be concluded that the elimination of gluten from the diet in people without diagnosed $C D$ does not improve the results of ART, while the results in people with CD improve significantly. It seems, therefore, that in women with no apparent cause of infertility, referred to ART, it is worthwhile to undergo screening tests for CD. Such an approach would avoid high financial and emotional costs in case of failure due to the presence of autoantibodies in the patient.

\section{NON-CELIAC WHEAT SENSITIVITY}

Recently, a condition that is Non-Celiac Wheat Sensitivity (NCWS) has been diagnosed more and more often. It is defined as the occurrence of gastrointestinal symptoms after wheat ingestion, without the presence of antibodies and intestinal villi atrophy, which are characteristics of CD, and without the presence of IgE specific to wheat allergy [31]. Currently, it is not known exactly what factor is responsible for the appearance of symptoms, hence the inconsistencies in the literature where both names can be found: Non-Celiac Wheat Sensitivity and Non-Celiac Gluten Sensitivity [32]. The diagnosis is made of exclusion, and the treatment is a GFD. No wonder then that when learning about this disease, the similarities and differences with CD are compared. For this reason, a study was developed to check the presence of gynaecological disorders in patients with NCWS. It showed more frequent occurrence of menstrual disorders, like polymenorrhea and oligomenorrhea in these patients [31]. However, an increase in the frequency of obstetric diseases and conditions were not observed. Because the condition itself is still poorly understood, its influence on the functioning of the female reproductive system cannot be ruled out with certainty. Even though there are no grounds for including NCWS in gynaecological diagnostics presently [31], it is worth being aware that such a disease exists. 


\section{GLUTEN-FREE DIET}

As it has been mentioned several times, the only effective treatment for $C D$ is the complete elimination of gluten from the diet [1]. Although, it may seem that since gluten itself does not play any specific role in the human body and giving up eating it should be completely safe, it turns out it is not the case.

A GFD introduced without earlier diagnosis of CD makes it impossible to confirm or exclude the existence of this condition in a patient [1]. If a patient decides to verify the correctness of this decision, the only option will be to return to a normal diet, and then perform diagnostics. This is a risky procedure, considering that if such a person has $C D$, then reintroducing gluten into the diet will result in autoantibody re-formation, intestine damage and recurrence of symptoms [33].

Another issue is the nuisance and higher cost of a GFD [33]. While the purchase of a single "gluten free" product is not a problem nowadays, controlling every purchased item or meal, also outside home, requires great self-discipline and is a real challenge. No wonder that people who, under the influence of information from the media, so eagerly decide to introduce a GFD, when they find out what it really looks like, give it up just as quickly.

Although in the case of people with CD, a GFD is essential and healthy, it should be mentioned that it is associated with the consumption of less fibre and more saturated fatty acids and products with a higher glycaemic index [33]. In addition, there are reports that gluten-free products are often low in Ca, Zn, Mg and Fe [34]. So, there is a kind of paradox here, that on one hand it is CD that, due to its pathomechanism, leads to deficiencies, and on the other its treatment, which is the elimination of gluten, also causes them.

Considering the above aspects, it can be safely stated that switching to a GFD, without the diagnosis of CD or other gluten-dependent disease, in order to improve fertility or avoid other gynaecological and obstetric disorders, may do more harm than good [33, 34]. Patients should be encouraged to carry out an early diagnosis, as well as to seek advice from specialists in the field of dietetics in cases where they want to obtain beneficial effects from the reproductive system through nutrition.

\section{SUMMARY}

Although research on the relationship between $C D$ and infertility often presents contradictory results, none of them has so far been carried out on a sufficiently large group of patients to constitute the basis for introducing recommendations. This contributes to the voluntary, not confirmed by research, transition of women with unexplained infertility to a GFD, often in an act of desperation when medicine becomes helpless for them. This diet, although generally safe, can generate deficiencies if not balanced, and may worsen fertility instead of improving it. Therefore, patients who consider gluten elimination from their diet should be encouraged to perform prior diagnosis of $C D$ and to consult a dietitian.

It is important that in the case of extraintestinal manifestation of $C D$, which causes the greatest diagnostic problem, suggestions can be found in the tests and the patient's history. These include the aforementioned iron deficiency anemia, delayed menarche, decreased AMH levels or a family history of $C D[1,12,20,21]$. At the moment it seems unreasonable to perform screening tests in all patients diagnosed with infertility, but for patients with one of the above-mentioned ones, it is worth considering.

It is also worth mentioning that some obstetric and gynaecological disorders are confirmed in women already diagnosed with $C D[7,14]$. Therefore, they should be additionally made aware of the importance of following a strict GFD in order to get pregnant and give birth to a healthy child. Moreover, if such a patient is referred to ART for various reasons, she must also know, that all kinds of dietary mistakes will reduce her chances of a positive result.

\section{Conflict of interest}

All authors declare no conflict of interest.

\section{REFERENCES}

1. Lebwohl B, Rubio-Tapia A. Epidemiology, presentation, and diagnosis of celiac disease. Gastroenterology. 2021; 160(1): 63-75, doi: 10.1053/j. gastro.2020.06.098, indexed in Pubmed: 32950520.

2. Wasilewski T, Łukaszewicz-Zając M, Wasilewska J, et al. Biochemistry of infertility. Clin Chim Acta. 2020; 508: 185-190, doi: 10.1016/j. cca.2020.05.039, indexed in Pubmed: 32446954.

3. Morris JS, Adjukiewicz AB, Read AE. Coeliac infertility: an indication for dietary gluten restriction? Lancet. 1970; 1(7640): 213-214, doi: 10.1016/s0140-6736(70)90572-6, indexed in Pubmed: 4189008.

4. Khizroeva J, Nalli C, Bitsadze V, et al. Infertility in women with systemic autoimmune diseases. Best Pract Res Clin Endocrinol Metab. 2019; 33(6): 101369, doi: 10.1016/j.beem.2019.101369, indexed in Pubmed: 31837981.

5. Sharif K, Watad A, Bridgewood C, et al. Insights into the autoimmune aspect of premature ovarian insufficiency. Best Pract Res Clin Endocrinol Metab. 2019; 33(6): 101323, doi: 10.1016/j.beem.2019.101323, indexed in Pubmed: 31606343.

6. Sen A, Kushnir VA, Barad DH, et al. Endocrine autoimmune diseases and female infertility. Nat Rev Endocrinol. 2014; 10(1): 37-50, doi: 10.1038/nrendo.2013.212, indexed in Pubmed: 24189508.

7. Casella G, Orfanotti G, Giacomantonio L, et al. Celiac disease and obstetrical-gynecological contribution. Gastroenterol Hepatol Bed Bench. 2016; 9(4): 241-249, indexed in Pubmed: 27895849.

8. Kreutz JM, Adriaanse MPM, van der Ploeg EMC, et al. Narrative review: nutrient deficiencies in adults and children with treated and untreated celiac disease. Nutrients. 2020; 12(2), doi: 10.3390/nu12020500, indexed in Pubmed: 32075276.

9. Grieger JA, Grzeskowiak LE, Wilson RL, et al. Maternal selenium, copper and zinc concentrations in early pregnancy, and the association with fertility. Nutrients. 2019; 11(7), doi: 10.3390/nu11071609, indexed in Pubmed: 31315178.

10. Fallah A, Mohammad-Hasani A, Colagar AH. Zinc is an essential element for male fertility: a review of $\mathrm{Zn}$ roles in men's health, germination, sperm 
quality, and fertilization. J Reprod Infertil. 2018; 19(2): 69-81, indexed in Pubmed: 30009140.

11. Michels KA, Wactawski-Wende J, Mills JL, et al. Folate, homocysteine and the ovarian cycle among healthy regularly menstruating women. Hum Reprod. 2017; 32(8): 1743-1750, doi: 10.1093/humrep/dex233, indexed in Pubmed: 28854586.

12. Mahadev S, Laszkowska M, Sundström J, et al. Prevalence of celiac disease in patients with iron deficiency anemia-a systematic review with meta-analysis. Gastroenterology. 2018; 155(2): 374-382.e1, doi: 10.1053/j.gastro.2018.04.016, indexed in Pubmed: 29689265.

13. Castaño M, Gómez-Gordo R, Cuevas D, et al. Systematic review and meta-analysis of prevalence of coeliac disease in women with infertility. Nutrients. 2019; 11(8), doi: 10.3390/nu11081950, indexed in Pubmed: 31434238.

14. Grode L, Bech BH, Plana-Ripoll O, et al. Reproductive life in women with celiac disease; a nationwide, population-based matched cohort study. Hum Reprod. 2018; 33(8): 1538-1547, doi: 10.1093/humrep/dey214, indexed in Pubmed: 29912336.

15. Gunn B, Murphy KE, Greenblatt EM. Unexplained infertility and undiagnosed celiac disease: study of a multiethnic canadian population. J Obstet Gynaecol Can. 2018; 40(3): 293-298, doi: 10.1016/j.jogc.2017.07.008, indexed in Pubmed: 29132967.

16. Grode LB, Agerholm IE, Humaidan $\mathrm{P}$, et al. Unrecognised coeliac disease among men and women undergoing fertility treatment: a screening study. United European Gastroenterol J. 2018; 6(10): 1477-1484, doi: 10.1177/2050640618796750, indexed in Pubmed: 30574318

17. Singh $P$, Arora A, Strand TA, et al. Diagnostic accuracy of point of care tests for diagnosing celiac disease: a systematic review and meta-analysis. J Clin Gastroenterol. 2019; 53(7): 535-542, doi: 10.1097/MCG.0000000000001081, indexed in Pubmed: 29912751.

18. Esteve $M$, Rosinach $M$, Llordés $M$, et al. Case-finding in primary care for coeliac disease: Accuracy and cost-effectiveness of a rapid point-of-care test. United European Gastroenterol J. 2018; 6(6): 855-865, doi: 10.1177/2050640618761700, indexed in Pubmed: 30023063.

19. Grode L, Møller Jensen T, Parkner T, et al. Diagnostic Accuracy of a Point-of-Care Test for Celiac Disease Antibody Screening among Infertile Patients. Inflammatory Intestinal Diseases. 2019; 4(3): 123-130, doi: 10.1159/000501520.

20. Kotze LM, Mallmann A, Miecznikowski RC, et al. Reproductive aspects in brazilian celiac women. Arq Gastroenterol. 2020; 57(1): 107-109, doi: 10.1590/S0004-2803.202000000-18, indexed in Pubmed: 32294744.

21. Cakmak E, Karakus S, Demirpence O, et al. Ovarian reserve assessment in celiac patients of reproductive age. Med Sci Monit. 2018; 24: 1152-1157, doi: 10.12659/msm.909033, indexed in Pubmed: 29476685.

22. Samsami A, Ghasmpour L, Moradi Alamdarloo S, et al. Women with Autoimmune Thyroiditis have Lower Reproductive Life Span or Not? A Cross- Sectional Study. Int J Community Based Nurs Midwifery.
2020; 8(4): 305-310, doi: 10.30476/ijcbnm.2020.84255.1207, indexed in Pubmed: 33178853.

23. Pereza N, Ostojić S, Kapović M, et al. A critical update on endothelial nitric oxide synthase gene variations in women with idiopathic recurrent spontaneous abortion: genetic association study, systematic review and meta-analyses. Mol Hum Reprod. 2015; 21(5): 466-478, doi: 10.1093/molehr/gav008, indexed in Pubmed: 25713339.

24. Anjum N, Baker PN, Robinson NJ, et al. Maternal celiac disease autoantibodies bind directly to syncytiotrophoblast and inhibit placental tissue transglutaminase activity. Reprod Biol Endocrinol. 2009; 7: 16, doi: 10.1186/1477-7827-7-16, indexed in Pubmed: 19228395.

25. Ajayi OO, Charles-Davies MA, Arinola OG. Progesterone, selected heavy metals and micronutrients in pregnant Nigerian women with a history of recurrent spontaneous abortion. Afr Health Sci. 2012; 12(2): 153-159, doi: 10.4314/ahs.v12i2.12, indexed in Pubmed: 23056021.

26. Thaker R, Oza H, Shaikh I, et al. Correlation of copper and zinc in spontaneous abortion. Int J Fertil Steril. 2019; 13(2): 97-101, doi: 10.22074/ijfs.2019.5586, indexed in Pubmed: 31037918.

27. Kucukaydin Z, Kurdoglu M, Kurdoglu Z, et al. Selected maternal, fetal and placental trace element and heavy metal and maternal vitamin levels in preterm deliveries with or without preterm premature rupture of membranes. J Obstet Gynaecol Res. 2018; 44(5): 880-889, doi: 10.1111/jog.13591, indexed in Pubmed: 29369445.

28. Alecsandru D, López-Palacios N, Castaño M, et al. Exploring undiagnosed celiac disease in women with recurrent reproductive failure: The gluten-free diet could improve reproductive outcomes. Am J Reprod Immunol. 2020; 83(2): e13209, doi: 10.1111/aji.13209, indexed in Pubmed: 31709662.

29. Juneau CR, Franasiak JM, Goodman LR, et al. Celiac disease is not more prevalent in patients undergoing in vitro fertilization and does not affect reproductive outcomes with or without treatment: a large prospective cohort study. Fertil Steril. 2018; 110(3): 437-442, doi: 10.1016/j.fertnstert.2018.03.030, indexed in Pubmed: 30098695.

30. Simopoulou M, Sfakianoudis K, Maziotis E, et al. The Impact of Autoantibodies on IVFTreatment and Outcome: A Systematic Review. Int J Mol Sci. 2019; 20(4), doi: 10.3390/ijms20040892, indexed in Pubmed: 30791371.

31. Soresi M, Incandela S, Mansueto P, et al. Gynecological disorders in patients with non-celiac wheat sensitivity. Dig Dis Sci. 2021;66(1): 167-174, doi: 10.1007/s10620-020-06184-8, indexed in Pubmed: 32146601.

32. Roszkowska A, Pawlicka M, Mroczek A, et al. Non-Celiac gluten sensitivity: a review. Medicina (Kaunas). 2019; 55(6), doi: 10.3390/medicina55060222, indexed in Pubmed: 31142014.

33. Diez-Sampedro A, Olenick M, Maltseva T, et al. A gluten-free diet, not an appropriate choice without a medical diagnosis. J Nutr Metab. 2019; 2019: 2438934, doi: 10.1155/2019/2438934, indexed in Pubmed: 31354988.

34. Rybicka I. The handbook of minerals on a gluten-free diet. Nutrients. 2018; 10(11), doi: 10.3390/nu10111683, indexed in Pubmed: 30400639. 